\title{
Measuring daily behavior using ambulatory accelerometry: The Activity Monitor
}

\author{
J. B. J. BUSSMANN \\ Erasmus University Rotterdam, Rotterdam, The Netherlands \\ W. L. J. MARTENS \\ PhyVision, Gemert, The Netherlands \\ J. H. M. TULEN \\ University Hospital Rotterdam, Rotterdam, The Netherlands \\ and \\ F. C. SCHASFOORT, H. J. G. VAN DEN BERG-EMONS, and H. J. STAM \\ Erasmus University Rotterdam, Rotterdam, The Netherlands
}

\begin{abstract}
Advanced ambulatory systems that measure aspects of overt human behavior during normal daily life have become feasible, owing to developments in data recording and sensor technology. One such instrument is the Activity Monitor (AM). This paper provides a technical description of the AM and information about its validity and current applications. The AM is based on ambulatory accelerometry, the aim of which is to assess postures and motions for long-term $(>24-\mathrm{h})$ measurement periods during normal daily life. Accelerometers are attached to the thighs, trunk, and lower arms, and signals are continuously stored in a digital portable recorder. In the postmeasurement analysis, postures and motions are detected by means of custom-made software programs. Validity studies performed on different populations showed high agreement scores between the computerized and automatic AM output and the visually analyzed video recordings. The AM has so far been applied in rehabilitation, psychophysiology, and cardiology but has many possibilities in behavioral research.
\end{abstract}

Human behavior and its measurement is important for such disciplines as medicine, physiotherapy, behavioral sciences, psychophysiology, and ergonomics. Overt human behavior can be regarded as a complex of postures and movements, including transitions between postures. Many techniques are used for the acquisition of data on (aspects of) postures and motions, including questionnaires, observations, diaries, kinetic, kinematic and video systems, actometers, and types of activity monitors (J. B. J. Bussmann \& Stam, 1998). However, most of these available techniques are inadequate for collecting the unobtrusive, objective, and valid measurements required to capture a large and specific set of postures and motions during normal daily life in a subject's personal environment.

Especially within the area of ambulatory systems, recent technological developments have led to advanced measurement systems (Busser, Ott, Uiterwaal, van Lum-

Correspondence concerning this article should be addressed to J. B. J. Bussmann, Department of Rehabilitation, University Hospital Rotterdam, P. O. Box 2040, 3000 CA Rotterdam, The Netherlands (e-mail: bussmann@ revd.azr.nl). mel, \& Blank, 1997; Fahrenberg, Foerster, Mueller, \& Smeja, 1997; Foerster \& Fahrenberg, 2000; Groeneveld, Waterlander, De Moel, Konijnendijk, \& Snijders, 1992; Kiani, Snijders, \& Gelsema, 1997; Uiterwaal, Glerum, Busser, \& van Lummel, 1998; Walker, Heslop, Plummer, Essex, \& Chandler, 1997). Small, portable, digital data logger systems have recently become available, allowing increased data processing and data storage capacities. Thanks to simultaneous developments in sensor technology, measurement during normal daily life is now feasible.

Although technology provides tools, is does not answer what should be measured or how it should be measured. The background of rehabilitation medicine (with its focus on the consequences of disease on daily and social functioning), the relevance of measuring postures and motions, and recent technological developments provided the rationale for developing the Activity Monitor (AM), an ambulatory instrument for measuring postures and motions during normal daily life (H. B. J. Bussmann, Reuvekamp, Veltink, Martens, \& Stam, 1998; J. B. J. Bussmann, Tulen, van Herel, \& Stam, 1998; J. B. J. Bussmann, van de Laar, Neeleman, \& Stam, 1998; Tulen, Bussmann, van Steenis, Pepplinkhuizen, \& Man in 't Veld, 1997; Tulen, Stronks, Bussmann, Pepplinkhuizen, \& 
Table 1

Segment, Position, and Sensitive Directions of the Accelerometers of the Activity Monitor, With the Subject in Anatomical Position

\begin{tabular}{|c|c|c|}
\hline Segment & Position & Sensitive Direction \\
\hline Thigh, right & $\begin{array}{l}\text { lateral side, halfway } \\
\text { between trochanter major } \\
\text { and knee joint }\end{array}$ & sagittal $(x)$ \\
\hline Thigh, left & $\begin{array}{l}\text { lateral side, halfway } \\
\text { between trochanter major } \\
\text { and knee joint }\end{array}$ & sagittal $(x)$ \\
\hline Trunk & lower part of sternum & sagittal $(x)$ \\
\hline Trunk & lower part of sternum & longitudinal $(z)$ \\
\hline Trunk & lower part of sternum & transverse $(y)$ \\
\hline Lower arm, right & distal part, lateral or dorsal & $\begin{array}{l}\text { sagittal }(x) \text { or } \\
\text { transverse }(y)\end{array}$ \\
\hline Lower arm, left & distal part, lateral or dorsal & $\begin{array}{l}\text { sagittal }(x) \text { or } \\
\text { transverse }(y)\end{array}$ \\
\hline
\end{tabular}

Passchier, 2000; van den Berg-Emons, Bussmann, Balk, $\&$ Stam, 2000). The postures of major interest were lying, sitting, and standing, and the motions were walking, running, climbing stairs, cycling, driving a wheelchair, noncyclic movements, and the transitions between different postures. Three aspects of these postures and motions can be distinguished: quantity (which posture/motion is performed, when, how often, and for how long), quality (how is the posture/motion performed), and physical strain (the physiological reaction of the body related to performing postures/motions).

The aim of this paper is to provide an up-to-date technical description of the AM. Although validity studies of the AM have been published, a comprehensive description was lacking. Furthermore, since these first studies, the analysis scheme and program of the AM have been changed and thoroughly extended. Another aim is to provide additional information about validity, applications, and general issues of ambulatory activity monitoring.

\section{TECHNICAL DESCRIPTION}

\section{Measurement Setup}

The standard configuration of the AM consists of four ADXL202 (Analog Devices, Breda, The Netherlands, adapted by Temec Instruments, Kerkrade, The Netherlands) piezo-resistive accelerometers (about $1.5 \times 1.5 \times 1$ $\mathrm{cm}$ ). The sensors are fixed on Rolian Kushionflex (Smith \& Nephew, Hoofddorp, The Netherlands) or siliconebased stickers (Schwa-Medico, Ehringshausen, Germany) by double-sided tape; both materials can be fixed directly on the skin. One sensor is attached at the lateral side of each thigh, at the level halfway between trochanter major and knee joint (Figure 1, Table 1). The sensitive axis of this uniaxial sensor is in a sagittal direction while subject is in the anatomical position. The third (biaxial) sensor is attached on the lower part of the sternum, with sensitive axes in the sagittal and longitudinal directions. At the sternum, an additional sensor with a sensitive transverse axis can be applied. In studies with a wheelchair-bound population or in studies aiming at measurement of arm-hand use, sensors are attached to the lower arm(s). These sensors can be additional or can be moved from the thighs or the trunk. All accelerometers have to be attached with their sensitive axis as parallel as possible to the related anatomical axis; a deviation of $15^{\circ}$ is allowed. If a sensor cannot be attached within this range, a wedge is used. Each accelerometer is attached to a data recorder by means of separate Lemo-jackets or with a multiconnector. This recorder is worn in a belt around the waist. Before measurements are started, the accelerometers are calibrated $(+1 \mathrm{~g},-1 \mathrm{~g})$.

\section{Piezo-Resistive Accelerometers}

Piezo-resistive accelerometers consist of a mass, connected to a frame by beams, which can be represented by a damped spring (Figure 2) (J. B. J. Bussmann, Damen, \& Stam, 2000; Veltink, Bussmann, de Vries, Martens, \& van Lummel, 1996; Winter, 1990). Piezo-resistors are

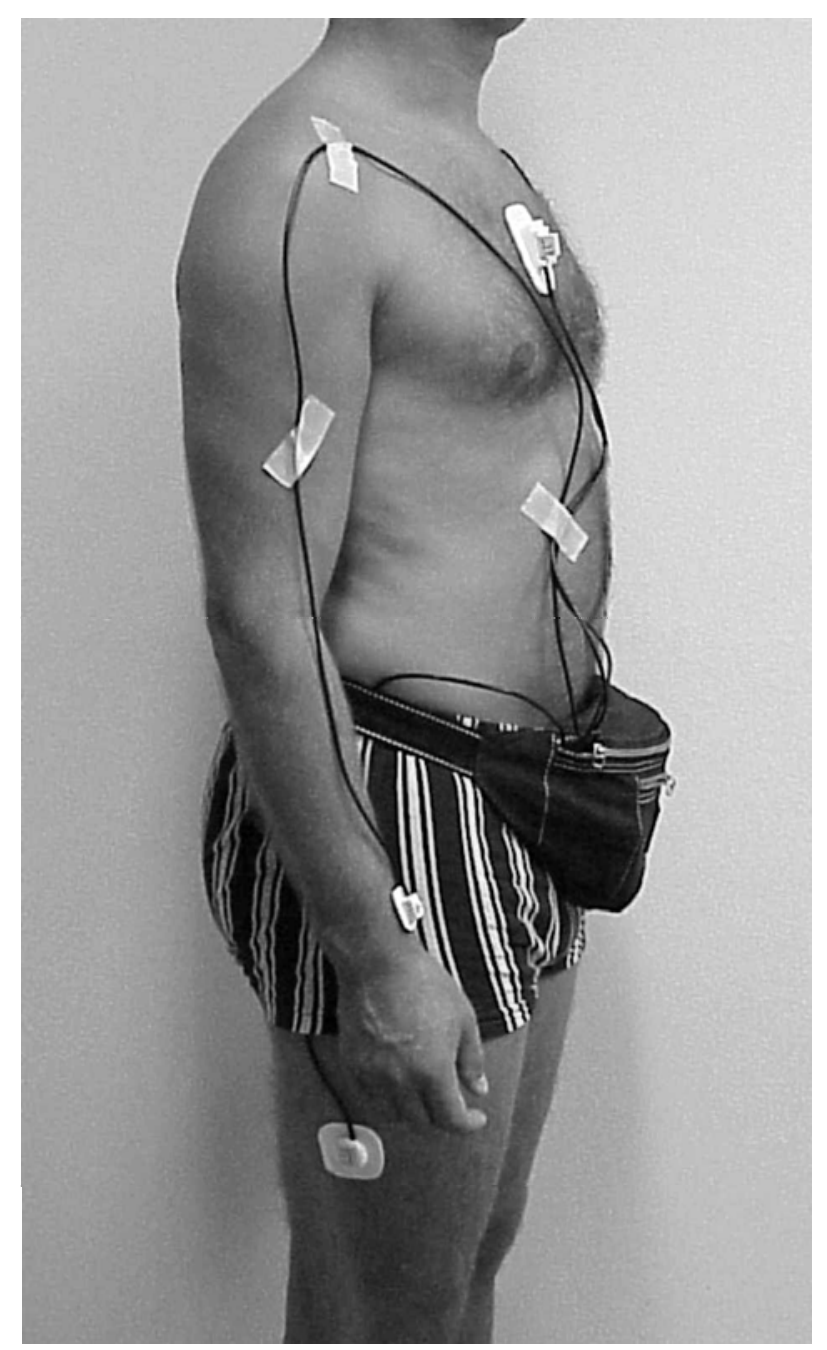

Figure 1. An extended configuration of the Activity Monitor, with accelerometers at the thighs, trunk, and lower arms. 


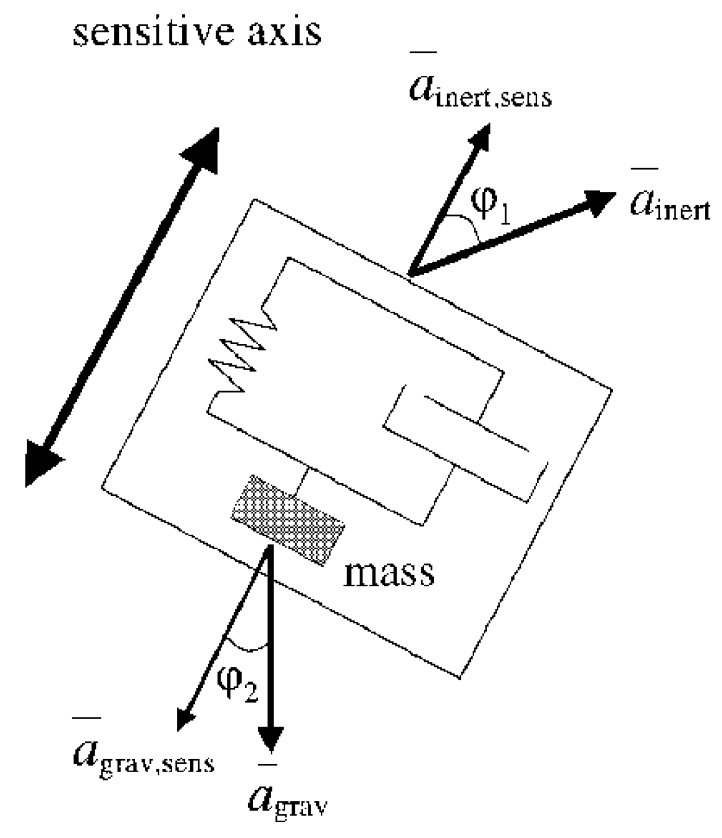

Figure 2. Scheme of a piezoresistive accelerometer. $\bar{a}_{\text {grav, }}$ gravitational acceleration; $\overline{\boldsymbol{a}}_{\text {inert, }}$ inertial acceleration; $\overline{\boldsymbol{a}}_{* * *, \text { sens, }}$, the part of the gravitational or inertial acceleration measured by the accelerometer.

mounted in the beams and form a bridge circuit. The value of the resistors depends on the magnitude of acceleration. The raw acceleration signals yielded by the piezoresistive accelerometers are a combination of a component of the gravitational acceleration $\left(\bar{a}_{\text {grav }}, 9.81 \mathrm{~m} / \mathrm{sec}^{2}\right)$ and a component of the inertial acceleration $\left(\bar{a}_{\text {inert }}\right)$. In static situations, the accelerometer signal yields only gravitational information, whereas in dynamic situations, this information is combined with inertial information (Morris, 1973; Padgoankar, Krieger, \& King, 1975; Veltink et al., 1996; Willemsen, van Alste, \& Boom, 1990). The part of $\bar{a}_{\text {grav }}$ that is measured ( $\left.\bar{a}_{\text {grav, sens }}\right)$ depends on the angle $\varphi_{2}$ between the sensitive axis of the sensor and $\bar{a}_{\text {grav }}$. The part of the $\bar{a}_{\text {inert }}$ that is measured ( $\left.\bar{a}_{\text {grav, sens }}\right)$ depends on the angle $\varphi_{1}$ between the sensitive axis of the sensor and $\bar{a}_{\text {inert }}$. A constant $\bar{a}_{\text {sens }}$ that is smaller than $9.81 \mathrm{~m} / \mathrm{sec}^{2}$ can be assumed to be the result of $\bar{a}_{\text {grav }}$. In this case, the angle between the accelerometer axis and the gravity vector can be determined from the accelerometer signal, which gives information about the orientation of the accelerometer.

\section{Recorder}

The type of data recorder is not crucial, although some requirements have to be met. The data logger should allow measurements for at least 1 day (data storage, energy supply), be able to measure (at least) four accelerometer signals, have small dimensions and low weight, and be easy to handle by researchers and clinicians. Currently, we use two recorder types. The Vitaport2 (Temec Instruments $)$ is a digital recorder $(9 \times 15 \times 4.5 \mathrm{~cm}, 700 \mathrm{gr})$, with its energy supplied by four penlite batteries. Its universal module allows simultaneous measurement of up to eight signals; data are stored on a flash card or a hard disk (with a memory capacity of up to $360 \mathrm{MB}$ ). Continuous measurement (without changing batteries or disks) over 3 days is possible. Recently, several prototypes of the Rotterdam Activity Monitor (or RAM, Temec Instruments) have been developed, which is a recorder that is based on Vitaport 2 technology but that is more dedicated to activity monitoring, allows up to four accelerometers, an ECG, and a marker signal, and is smaller and lighter $(9 \times 15 \times 3.5 \mathrm{~cm}, 500 \mathrm{gr})$ than the Vitaport 2 .

The data recorder must contain a definitionfile, which contains the measurement setup. The measurement setup consists (among other things) of calibration and offset factors, sample frequency $(32 \mathrm{~Hz})$, resolution (12 bits), and filters (30-Hz low-pass). Signals are continuously measured and stored. After the measurements, the data are downloaded onto a PC for analysis.

\section{Analysis}

After the measurements, automatic data analysis takes place by means of a proprietary signal processing and inferencing language (S.P.I.L.), yielding C-code (Jain, Martens, Mutz, Weiss, \& Stephan, 1996). In this automatic analysis, three parts can be distinguished(Figure 3): (1) feature extraction (i.e., new signals [feature signals] with specific characteristics are derived and computed from each measured signal), (2) posture/motion detection (i.e., on the basis of the feature signals, postures and motions are classified), and (3) postprocessing (i.e., output signals of the posture/motion detection are processed so that readable and relevant information is provided). For a 48-h measurement, Parts 1 and 2 take about $30 \mathrm{~min}$. The three parts are discussed in the following sections.

Feature extraction. For posture/motion detection, three feature signals are derived and continuously computed from each measured accelerometer signal (Figure 4).

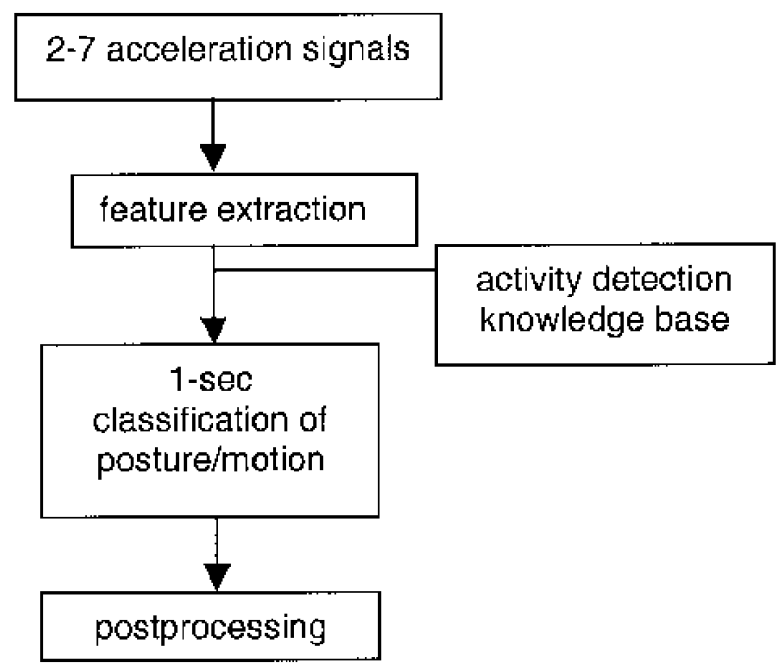

Figure 3. General analysis scheme. 
Table 2

Subcategories (Postures and Motions) of the Activity Monitor

\begin{tabular}{cc}
\hline AM Subcategory & Remark \\
\hline
\end{tabular}

Lying prone, standard

Lying prone, trunk slightly raised

Lying on side, trunk slightly forward

Lying on side, trunk slightly backward

Lying on side, trunk strongly forward

Lying on side, trunk strongly backward

Lying supine

Standing, standard

Standing, trunk slightly flexed

Standing, trunk strongly flexed

Sitting, standard

Sitting, trunk backward

Sitting, trunk slightly flexed

Sitting, trunk strongly flexed

General movement

Walking, standard

Walking, slow

Walking, fast

Climbing stairs, down

Climbing stairs, up

Cycling

Driving a wheelchair

right/left optional right/left optional right/left optional right/left optional

noncyclical movement

Running

currently by means of postprocessing

1. Low pass/angular feature. The LP/angular signals are created after low-pass filtering (finite impulse response filter, cutoff frequency, $0.3 \mathrm{~Hz}$ ). The signal is subsequently averaged over 1 -sec intervals and converted to angles via an arcsine transformation (range, $-90^{\circ}$ to $+90^{\circ}$ ), although the translation to an angular position of the sensor is not straightforward (e.g., during motion and multidimensional angular positions).

2. Motility feature. The motility signals are created after subsequent high-pass filtering at $0.3 \mathrm{~Hz}$, rectifying, and averaging. The high-pass-filtered derivative is actually effectuated by subtracting the low-pass-filtered signal (see LP/angular feature) from the raw measured signal. During a $1-\mathrm{sec}$ interval, a fixed window of data is averaged; the mean value is assigned to the motility signal $(1 \mathrm{~Hz})$. This mean value depends on the variability of the measured signal around the mean and can be considered as acceleration energy.

3. Frequency feature. The frequency signals are based on a band-pass-filtered derivative $(0.3-2 \mathrm{~Hz}$ for the legs and lower arms, 0.6-4 Hz for the trunk), also with the use of finite impulse response filters. This band-passed signal (which ideally has a sinusoid shape with the movement frequency of the segment the sensor is attached to) is the input of the fast time frequency transform (FTFT) procedure (Martens, 1992). This procedure constitutes a particular type of instantaneous frequency analysis for signals and determines instantaneously the frequency and amplitude/envelope of the band-passed signal. To be regarded as valid, this raw instantaneous frequency must meet three preset criteria in the so-called FTFT knowledge base: the frequency range, the amplitude (power)

range of the band-pass-filtered signal, and the variability of the detected frequency. If the current signal does not meet all the preset criteria, no valid frequency is assigned; otherwise, the frequency is assigned to a frequency signal and averaged over 1-sec intervals.

Posture/motion detection. In the analysis program, more than 20 (sub)postures and motions are distinguished (Table 2). Detection of these posture/motion subcategories is based on the feature signals, derived from the measured signals. For each subcategory and for each feature signal, a minimum and maximum value is preset in the activity detection knowledge base. For consecutive moments in time ( $1 \mathrm{sec})$, for each subcategory and for each feature signal, the distance is calculated from the actual feature signal value to the preset range. Since the three features (LP/angular, motility, and frequency) have different units (degrees, $g$, and $\mathrm{Hz}$, respectively), some features are scaled to allow a proportional influence of all features. If an actual feature signal value is within the preset range of a specific posture/motion subcategory, the calculated distance is zero-that is, it does not add to the total distance for that subcategory. The calculated distances of the feature signals are added for each subcategory; the posture/motion subcategory with the lowest total distance in the end will be selected and detected (Figure 5). If a posture/motion is detected but the distance is above a preset general threshold, indicating a relatively high degree of unreliability, the category unknown is selected.

Postprocessing. After the posture/motion detection, there are some (optional) postprocessing procedures.

1. From subcategories to main categories. Although most of the 23 posture/motion subcategories are required initially to avoid misdetection, not all 23 subcategories have to be of interest in a later phase. Reducing the number of categories (by combining some postures/motions) may be desirable, depending on the application of interest. In our studies, the subcategories were reduced to the main categories: the postures of lying, sitting, and standing and the motions of walking, running, climbing stairs, cycling, driving a wheelchair, noncyclic movements, and the transitions between different postures.

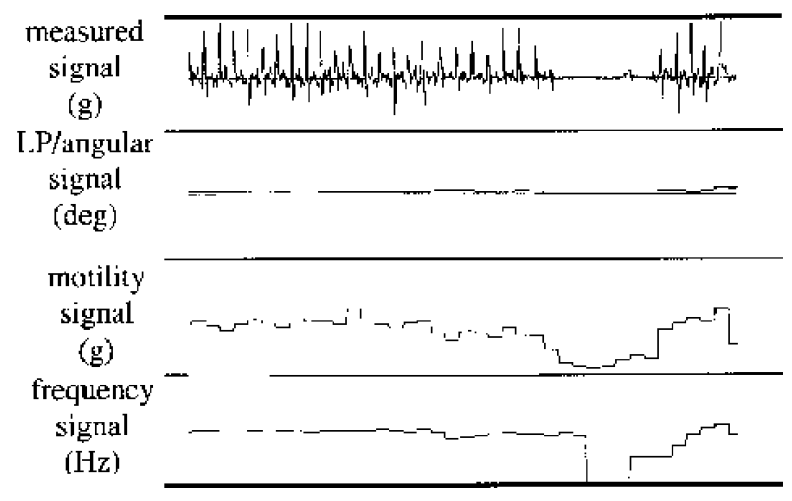

Figure 4. A measured thigh signal and its three feature signals (LP/angular, motility, and frequency). 

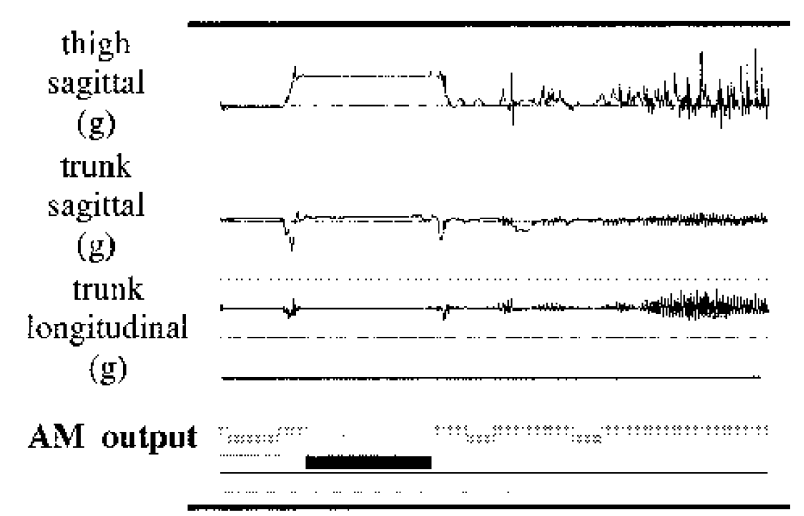

Figure 5. Input (three measured signals: one thigh, two trunk) and output (automatic posture/motion detection) of the Activity Monitor (AM) data analysis program (upper/gray bars, transition/walking; middle/gray bars, standing; lower/black bar, sitting).

2. Duration threshold. Each second a posture/motion is selected. The advantage of this high temporal resolution is that it allows tracing of short-lasting postures/motions (e.g., walking a few steps and consecutive transitions in a short time). However, this high resolution also has two disadvantages. First, error detections are mostly of short duration and thus will be present if a 1-sec resolution is used. Second, (very) short-lasting postures/ motions may not be of clinical or methodological interest. For this reason, a postprocessing procedure is included by which postures/motions below a certain duration are rejected. In fact, each sample within a time window of $x$ sec around sample $s$ is examined; the posture/motion that is most frequently detected in that window is assigned to sample $s$. The size of the frame determines the duration threshold. In our studies, a threshold of $5 \mathrm{sec}$ is applied.

3. Manual editing. Although the validity of AM measurements is high (see the Validity Studies section), at present not all postures/motions are detected or correctly detected. For example, it may be desirable to distinguish driving a car (which can be determined by visual inspection but is not yet an automated output category) from sitting. In such situations, it is possible to manually correct or insert categories.

4. Statistics/reports. Several output measures can be derived from the posture/motion detection; the measures discussed in this paragraph are just a few examples of several options. For each posture/motion, the total duration can be calculated. Furthermore, per posture/motion, a frequency histogram can be made, with duration categories on the $x$-axis (e.g., $0-10 \mathrm{sec}, 10-30 \mathrm{sec}$, etc.). The number of transitions between different postures can be calculated and displayed in an $n \times n$ table. Motility signals can also be used to provide outcome measures; generally, the two trunk and two thigh motility signals are averaged to calculate the body motility. This measure is assumed to be related to the overall level of physical ac- tivity during the measurement. Body motility during walking is a measure of walking speed. Furthermore, heart rate data can be combined with posture/motion categories-for example, mean heart rate can be computed for each posture/motion category. The parameters may comprise the whole measurement period or one or more parts of it.

\section{VALIDITY STUDIES}

The validity of the Activity Monitor has been studied extensively (H. B. J. Bussmann et al., 1998; J. B. J. Bussmann, Tulen, et al., 1998; J. B. J. Bussmann, van de Laar, et al., 1998; van den Berg-Emons et al., 2000). In these four studies, able-bodied subjects, persons with a leg amputation, students participating in a psychophysiological study, failed back surgery patients, and chronic heart failure patients were involved. Subjects generally performed a large number of functional activities in their own manner and pace. Measurements were performed with the AM, and simultaneously videotape recordings were made. In these papers-except that of van den Berg-Emons et al. (2000) - a previous version of the AM was described. This first version of the AM (identical in sensor configuration, but different in analysis program) allowed one to distinguish several postures (standing, sitting, and different forms of lying) and motions (e.g., walking, climbing stairs, cycling) as one group. Motions were distinguished from postures on the basis of one Boolean algorithm, the posture/motion detection procedure was hierarchical, and several motions could not be distinguished from each other. Because the current updated AM differs from the first AM version only in its analysis program, the data from the original analysis can be used for validation of the current AM version. Validity was assessed by calculating agreement scores between AM output and video output and by comparing the number of walking periods and the duration of postures/motions determined by both methods.

The overall agreement between AM output (current version) and videotape analysis for the four studies was $89 \%, 93 \%, 81 \%$, and $90 \%$ (J. B. J. Bussmann, 1998; van den Berg-Emons et al., 2000). In the three studies with dynamic periods of considerable duration, walking had agreement scores per recording ranging from $67 \%$ to $95 \%$. Differences from the videotape analysis occurred mostly in gray areas, such as shuffling and standing with movement. For climbing stairs, the sensitivity scores were somewhat lower (overall mean per study, $24 \%, 76 \%$, and $49 \%$; range, $0-87 \%$ ), generally owing to the misdetection of walking. Generally, the duration of walking was slightly underestimated $(-0.8 \%,-0.8 \%$, and $-4.1 \%)$. The number of walking periods was well determined (total number, 169 vs. 170,255 vs. 240 , and 94 vs. 110 , respectively). The agreement score per measurement for cycling ranged from $51 \%$ to $100 \%$.

It can be concluded that the current version of the AM is a valuable extension of the first AM version. The de- 


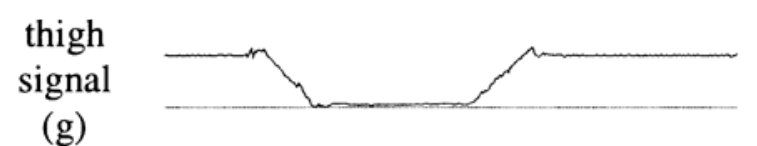

(g)

trunk

signal

(g)

Figure 6. Measured sagittal thigh and sagittal trunk signal during the sit-to-stand transition and stand-to-sit transition.

tection of postures remained stable, and walking and cycling were well determined. The ability to distinguish climbing stairs was less powerful, but a more valid determination of angular attachment deviations may improve this. Currently, an ongoing study is aimed at the validity of the detection of driving a wheelchair, with additional accelerometers attached at the lower arms.

\section{CURRENT APPLICATIONS}

Daily behavior expressed as postures and motions is important for both experimental and clinical research, making use of the AM in descriptive, explorative, and evaluative studies relevant. Because the authors are involved mainly with rehabilitation and psychophysiology, the following examples of AM applications are limited to their field of expertise only.

The AM is evaluatively used in a randomized clinical trial (RCT) focused on the effect of aerobic training on daily activity in heart failure patients. Other RCTs concern the effects of specific drugs on fatigue and activity pattern in postpolio patients, the effect of a novel socket on daily physical activity in persons with a lower leg amputation, and the effects of drugs on daily functioning in migraine patients. In a previous stage, the effect of benzodiazepines on physical activity was studied (Tulen et al., 1997). The AM is also used in a pilot study focused on the effect of exercise training on daily physical activity in Guillain-Barré patients. Examples of the use of the AM in descriptive and explorative research are two studies on the extent of hypoactivity in patients with meningomyelocele and cerebral palsy, a study on the determinants of daily physical activity in meningomyelocele patients, a study on the effects of a multidisciplinary treatment program in chronic pain patients, and a study on the recovery of physical activity in spinal cord injury patients. In another study, the AM is used for the objective measurement of arm-hand use in patients with reflex sympathetic dystrophy.

These are just a few examples; the reader may extrapolate the possibilities to his or her own field of research.

\section{QUALITY AND PHYSICAL STRAIN}

Apart from the quantity aspects of postures and motions, there are quality and physical strain aspects.

\section{Quality}

The acceleration signals contain valuable information about quality of postures and motions-that is, movement pattern, coordination, speed, and so on. (J. B. J. Bussmann, Damen, \& Stam, 2000; Willemsen et al., 1990). In our research, walking and the sit-to-stand transition are of special interest. In walking, such parameters as stride frequency, walking speed, symmetry, and stability can be derived from the acceleration signals. For sit-to-stand transitions, such parameters as movement time, movement pattern, and phasing of leg and trunk movements are considered important (Figure 6). Many quality aspects still need to be explored.

\section{Physical Strain}

Acceleration signal-based parameters are often used in the long-term measurement of energy or oxygen consumption. The body motility signal (derived from the motility signals used for the detection of postures and motions) appeared to have a close relationship with oxygen uptake and heart rate during walking (Figure 7); (J. B. J. Bussmann, Hartgerink, van der Woude, \& Stam, 2000). Although recent studies show a more complex picture when motility is used as a physical strain measure in persons with different movement efficiency and in motions other than walking, the use of motility may increase the validity of ambulatory long-term measurement of physical strain.

\section{DISCUSSION}

\section{Future Developments}

The development of an instrument such as the AM is an ongoing process of extending possibilities and optimizing current properties. Thus, current and future develop-

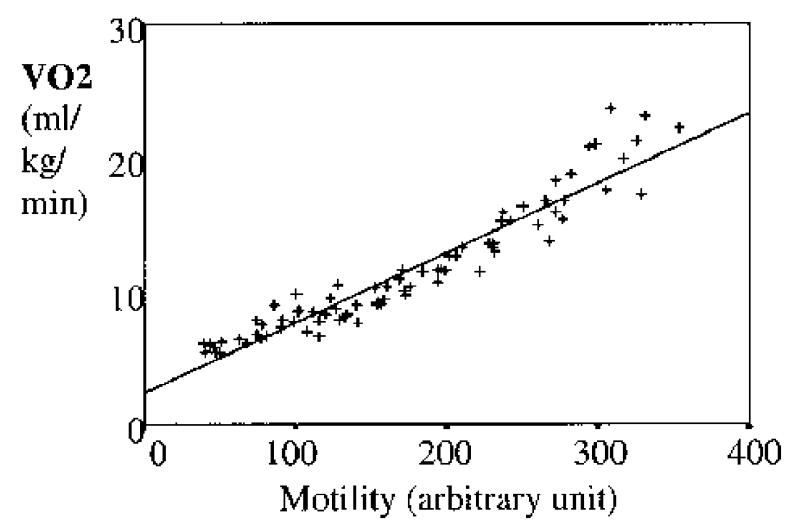

Figure 7. Relation between body motility (as measured with the $A M$ ) and oxygen uptake during walking (data from 12 ablebodied subjects, walking at fixed speeds from 0.8 to $7.2 \mathrm{~km} / \mathrm{h}$, with $0.8-\mathrm{km} / \mathrm{h}$ intervals. 
ments mainly concern the topics described here- that is, the validity of posture/motion detection (e.g., optimization of the detection of climbing stairs and wheelchair driving), the use in (evaluative) studies, and the measurement of quality and physical strain. An important extension, however, is the additional use of accelerometers attached at the (lower) arms for the ambulatory measurement of arm-hand use and for the detection of wheelchair driving.

For the sensor and recorder configuration, future technological developments should focus on a smaller and lighter recorder and sensors, and wireless connections between sensors and recorder may be a promising option.

In most of our research, accelerometer and ECG signals are standard. The acceleration signals can, however, be combined with the simultaneous measurement of other signals, such as blood pressure, EMG, temperature, and so forth.

\section{Methodological, Practical, and Ethical Issues}

Use of the AM in research presents some methodological concerns. First, some studies show a considerable between-days variability in activity pattern, which has consequences for group size and number of measurement days. Second, external factors (e.g., weather and season) may influence the activity pattern, which may raise problems in (longitudinal) studies without control groups. Third, the AM may influence the activity pattern of subjects: Patients are aware that they are measured (reactivity effect or perturbation effect; Fahrenberg, 1996), and use of the AM may prevent the performance of some activities (e.g., the AM can be used during sports, but not during showering, which may prevent the person from exercising). To address the reactivity effect, we usually have an agreement with the patients that we will inform them only after measurement. Regarding the hindrance to activities, optimization of sensor attachment (e.g., allowing the person to attach/remove the sensors) may be a solution.

Costs are another unavoidable factor. Owing to production in small batches, the cost of a complete system is currently about $\$ 10,000$; a current economic dilemma is the investment needed for large-scale production leading to a substantially lower unit price.

Research with the AM is subject to the usual guidelines of medical and ethical committees. The AM could be perceived by some as "Big Brother is watching you"- - that is, as an invasion of one's privacy. It should be specifically mentioned to all patients that the AM provides a set of postures/motions only. The output is no more and no less than these output categories.

\section{Comparison With Other Activity Monitors}

The concept of ambulatory measurement of postures and motion is not new. Mechanical movement counters (Davies, Jordan, \& Lipkin, 1992; Saris \& Binkhorst, 1977), EMG (Anastasiades \& Johnston, 1990; Tuomisto, Johnston, \& Schmidt, 1996), hydrostatic tubes (Tuomisto et al., 1996), gyroscopes (Miyazaki, 1997), and accelerometers (Stock, Clague, \& Johnston, 1991; Tuomisto et al., 1996) have been used for this purpose. Generally, comparison of the AM with these monitoring systems is difficult. Instruments discriminate different posture/motion categories, they are used for different aims, or validity is determined by following a different or unknown method. The number of instruments that resemble the characteristics of the AM is small. In the literature, only a few systems are reported that are aimed at a similar set of postures/motions and that are based on accelerometry.

Walker and colleagues (Walker et al., 1997) reported a validation study of an activity monitor based on mercury switches and accelerometers. Posture and the number and vigour of steps were recorded. Validation was studied in terms of steps counted; validation of body positions was not reported. In that study, the relationship between activity and disability was explored.

Kiani and colleagues (Kiani et al., 1997, 1998) and Groeneveld and collegues (Groeneveld et al., 1992) described the AMMA system, an activity monitor with the analysis initially based on a neural network. Although a neural network may be a powerful tool, it has the disadvantages that it needs training data and has an extended analysis time. Kiani and collegues (Kiani et al., 1998) also proposed and tested a fuzzy logic type of analysis, which comes close to the type of analysis of the AM. Although validation results of their system are presented, the way the results were obtained was not clearly described, and therefore, the results difficult to interpret.

Another instrument similar to the AM is the Dynaport ADL monitor described by Busser et al. (1997) and Uiterwaal et al. (1998). In the Dynaport system, the trunk sensors are integrated into the recorder, which is carried in a belt around the waist. The instrument has been validated in children and during working conditions; an overall agreement percentage of $76 \%-93 \%$ was reported. It is not clear how postures/motions are distinguished in the analysis, but the posture detection is probably based on the same principle as that used in the AM.

Fahrenberg and collegues (Fahrenberg et al., 1997) and Foerster and Fahrenberg (2000) studied the possibilities of accelerometry to detect postures and motions from a psychophysiological viewpoint, also using a Vitaport measurement system. Fahrenberg and colleagues applied accelerometers and a hydrostatic tube to monitor their subjects' ambulatory activity. In the study of Foerster and Fahrenberg, the validity of a reference-patternbased classification and a hierarchical strategy was studied, using accelerometer signals as input. In such a method, individual reference patterns for each type of posture and movement are obtained from an initial reference recording. Therefore, this method is different from the fixed-threshold classification method of the AM. The main disadvantage of the fixed-threshold method is its potential sensitivity to interindividual differences in performing postures/motions and to differences in sensor position between subjects and between measurements, whereas the main advantage is its transparency and the fact that no initial reference recording is needed. Although only a small number (13) of standardized postures/motions were performed in the study of 
Foerster and Fahrenberg, the reliability of posture and motion detection was high (3.2\% misclassifications).

\section{Conclusion}

The AM is or has been applied in research related to rehabilitation medicine, psychophysiology, and cardiology. However, its possibilities are not limited to these disciplines. Ambulatory monitoring of the quantity of postures and motions is also relevant in the behavioral sciences. This relevance will be enhanced by the possibility of combining posture/motion monitoring with the simultaneous measurement of other signals (such as EMG) and by exploring the features contained in the accelerometer signal, such as motility, frequency, stability over time, and phasing, that are associated with quality.

\section{REFERENCES}

Anastasiades, P., \& Johnston, D. W. (1990). A simple activity measure for use with ambulatory subjects. Psychophysiology, 27, 87-93.

Busser, H. J., Ott, J., UiterwaAl, M., van, Lummel, R. C., \& Blank, R. (1997). Ambulatory monitoring of children's activity. Medical Engineering \& Physics, 19, 440-445.

Bussmann, H. B. J., Reuvekamp, P. J., Veltink, P. H., Martens, W. L. J., \& STAM, H. J. (1998). Validity and reliability of measurements obtained with an "Activity Monitor" in people with and without a transtibial amputation. Physical Therapy, 78, 989-998.

BussmanN, J. B. J. (1998). Ambulatory monitoring of mobility-related activities in rehabilitation medicine. Delft, The Netherlands: Eburon.

Bussmann, J. B. J., Damen, L., \& Stam, H. J. (2000). Analysis and decomposition of signals obtained by thigh-fixed accelerometry during walking. Medical \& BiologicalEngineering \& Computing, 38, 632-638.

Bussmann, J. B. J., Hartgerink, I., van der Woude, L. H. V., \& STAM, H. J. (2000). Measuring physical strain during ambulation with accelerometry. Medicine \& Science in Sports \& Exercise, 32, 1462-1471.

Bussmann, J. B. J., \& Stam, H. J. (1998). Techniques for measurement and assessment of mobility in rehabilitation: A theoretical approach. Clinical Rehabilitation, 12, 455-464.

Bussmann, J. B. J., Tulen, J. H. M., van Herel, E. C. G., \& Stam, H. J. (1998). Quantification of physical activities by means of accelerometry: A validation study. Psychophysiology, 35, 488-496.

Bussmann, J. B. J., van de Laar, Y. M., Neeleman, M. P., \& Stam, H. J. (1998). Ambulatory accelerometry to quantify motor behavior in patients after failed back surgery. Pain, 74, 153-161.

Davies, S. W., Jordan, S. L., \& Lipkin, D. P. (1992). Use of limb movement sensors as indicators of the level of everyday physical activity in chronic congestive heart failure. American Journal of Cardiology, 69, 1581-1586.

FAHRENBERG, J. (1996). Ambulatory assessment: Issues and perspectives. In J. Fahrenberg \& M. Myrtek (Eds.), Ambulatory assessment: Computer-assisted psychological and psychophysiological methods in monitoring and field studies (pp. 3-20). Seattle: Hogrefe \& Huber.

Fahrenberg, J., Foerster, F., Mueller, W., \& Smeja, M. (1997). Assessment of posture and motion by multi-channel piezoresistive accelerometer recordings. Psychophysiology, 34, 607-612.

Foerster, F., \& Fahrenberg, J. (2000). Motion pattern and posture: Correctly assessed by calibrated accelerometers. Behavior Research Methods, Instruments, \& Computers, 32, 450-457.

Groeneveld, W. H., Waterlander, K. J., De Moel, A. V. L., KoniJNENDIJK, H. J., \& SNIJDERS, C. J. (1992). Instrumentation for ambulatory monitoring of patient movement. In Proceedings of the 12th International Symposium on Biotelemetry (pp. 558-564).

Jain, A., Martens, W. L. J., Mutz, G., Weiss, R. K., \& Stephan, E.
(1996). Towards a comprehensive technology for recording and analysis of multiple physiological parameters within their behavioral and environmental context. In J. Fahrenberg \& M. Myrtek (Eds.), Ambulatory assessment: Computer-assisted psychological and psychophysiological methods in monitoring and field studies (pp. 215 236). Seattle: Hogrefe \& Huber.

Kiani, K., Snijders, C. J., \& Gelsema, E. S. (1997). Computerized analysis of daily life motor activity for ambulatory monitoring. Technology \& Health Care, 5, 307-318.

Kiani, K., Snijders, C. J., \& Gelsema, E. S. (1998). Recognition of daily motor activity classes using an artificial neural network. Archives of Physical Medicine \& Rehabilitation, 79, 147-154.

Martens, W. L. J. (1992). The Fast Time Frequency Transform (F.T.F.T.): A novel approach to the Instantaneous Spectrum. In Proceedings of the 14th International Conference of the IEEE Engineering in Medicine \& Biology Society. Piscataway, NJ: IEEE Press.

MiYAZAKI, S. (1997). Long-term unrestrained measurement of stride length and walking velocity utilizing a piezoelectric gyroscope. IEEE Transactions on Biomedical Engineering, 44, 753-759.

Morris, J. R. W. (1973). Accelerometry: A technique for the measurement of human body movements. Journal of Biomechanics, 6, 729-736.

Padgoankar, A. J., Krieger, K. W., \& King, A. I. (1975). Measurement of angular acceleration of a rigid body using linear accelerometers. Journal of Applied Mechanics, 42, 552-556.

SARIS, W. H. M., \& Binkhorst, R. A. (1977). The use of pedometer and actometer in studying daily physical activity in man: Pt. 1. Reliability of pedometer and actometer. European Journal of Applied Physiology, 37, 219-228.

Stock, S. E., Clague, M. B., \& Johnston, I. D. A. (1991). Postoperative fatigue: A real phenomenon attributable to the metabolic effects of surgery on body nutritional stores. Clinical Nutrition, 10, 151-157.

Tulen, J. H. M., Bussmann, J. B. J., van Steenis, H. G., PeppLinkHuizen,L., \& MAN IN 'T Veld, A. J. (1997). A novel tool to quantify physical activities: Ambulatory accelerometry in psychopharmacology. Journal of Clinical Psychopharmacology, 17, 202-207.

Tulen, J. H. M., Stronks, D., Bussmann, J. B. J., Pepplinkhuizen, L., \& PAsschier, J. (2000). Towards an objective quantitative assessment of daily functioning in migraine: A feasibility study. Pain, 86, 139-149.

Tuomisto, M. T., Johnston, D. W., \& Schmidt, T. F. H. (1996). The ambulatory measurement of posture, thigh acceleration and muscle tension and their relationship to heart rate. Psychophysiology, 33, 409-415.

UiterwaAl, M., Glerum, E. B., Busser, H. J., \& van Lummel, R. C. (1998). Ambulatory monitoring of physical activity in working situations, a validation study. Journal of Medical Engineering \& Technology, 22, 168-172.

van den Berg-Emons, R. J. G., Bussmann, J. B. J., Balk, A. H. M. M., \& STAM, H. (2000). Validity of ambulatory accelerometry to quantify physical activity in heart failure. Scandinavian Journal of Rehabilitation Medicine, 32, 187-192.

Veltink, P. H., Bussmann, H. B. J., de Vries, W., Martens, W. L. J., \& VAN Lummel, R. C. (1996). Detection of static and dynamic activities using uniaxial accelerometers. IEEE Rehabilitation Engineering, 4, 375-385.

Walker, D. J., Heslop, P. S., Plummer, C. J., Essex, T., \& Chandler, S. (1997). A continuous patient activity monitor: Validation and relation to disability. Physiological Measurement, 18, 49-59.

Willemsen, A. T. M., van Alste, J. A., \& Boom, H. B. K. (1990). Real-time gait analysis utilizing a new way of accelerometry. Journal of Biomechanics, 23, 859-863.

WinTER, D. A. (1990). Biomechanics and motor control of human movement. New York: Wiley.

(Manuscript received October 6, 2000; revision accepted for publication June 12, 2001.) 